\title{
Neutrino Self-Interactions and XENON1T Electron Recoil Excess
}

\author{
Andreas Bally๑, ${ }^{*}$ Sudip Jana๑, ${ }^{\dagger}$ and Andreas Trautner $\odot^{\ddagger}$ \\ Max-Planck-Institut für Kernphysik, Saupfercheckweg 1, 69117 Heidelberg, Germany
}

(Received 23 June 2020; accepted 9 September 2020; published 12 October 2020)

\begin{abstract}
The XENON1T collaboration recently reported an excess in electron recoil events in the energy range between 1-7 keV. This excess could be understood to originate from the known solar neutrino flux if neutrinos couple to a light vector mediator with strength $g_{\nu N}$ that kinetically mixes with the photon with strength $\chi$ and $g_{\nu N} \chi \sim 10^{-13}$. Here, we show that such coupling values can naturally arise in a renormalizable model of long-range vector-mediated neutrino self-interactions. The model could be distinguished from other explanations of the XENON1T excess by the characteristic $1 / T^{2}$ energy dependence of the neutrino-electron scattering cross section. Other signatures include invisible Higgs and $Z$ decays and leptophilic charged Higgses at a few $100 \mathrm{GeV}$. ALPS II will probe part of the viable parameter space.
\end{abstract}

DOI: 10.1103/PhysRevLett.125.161802

A recently publicized search for low-energy electronic recoil events performed with the XENON1T detector yielded some unexpected excess over background that was statistically significant at the level of $3.2 \sigma$ [1]. New physics explanations of this excess could include axions produced in the Sun [1], an unexpectedly sizable neutrino magnetic moment [1], inelastic semi-annihilation recoils [2], axionlike particle warm dark matter [2], or a fast dark matter component [3]. The solar axion and neutrino magnetic moment explanations are already practically ruled out, respectively, by stellar cooling [4] or white dwarfs [5] and globular cluster cooling constraints [6,7]. A more conventional origin of the excess would be an unaccounted Tritium background in the detector [1] or simply a statistical fluctuation. Notwithstanding this perception, here we proclaim a new physics explanation of the excess based on a UV-complete neutrino selfinteraction model recently proposed by Berbig, Jana, and Trautner [8].

Elastic scattering of solar neutrinos off electrons is a subdominant background for low-energy electron recoil events in XENON1T with around 220 expected events [1]. The inclusion of a light mediator that couples neutrinos to electrons could change this conclusion and explain the observed excess, just as in the case of an enhanced neutrino magnetic moment. However, generic light mediators, especially when coupled to electrons, face severe constraints (see, e.g., [9-11]). As in [8], we circumvent

Published by the American Physical Society under the terms of the Creative Commons Attribution 4.0 International license. Further distribution of this work must maintain attribution to the author(s) and the published article's title, journal citation, and DOI. Funded by SCOAP ${ }^{3}$. the most rigorous constraints from cosmology by relying on a low temperature phase transition in the neutrino sector [12]. However, different from [8], where mediator masses of $m_{Z^{\prime}} \sim \mathcal{O}(10) \mathrm{eV}$ were considered, we here focus on a parameter region with $m_{Z^{\prime}} \lesssim 10^{-4} \mathrm{eV}$. The reason is that in order to accommodate the observed excess at XENON1T, there needs to be a sufficiently strong interaction between electrons and the new mediator, which is excluded for the former region. In the new parameter region, the phase transition after which neutrinos start mixing with the hidden sector happens after recombination, i.e., below $T \sim 1 \mathrm{eV}$.

The UV-complete and renormalizable model has been presented in some detail in [8], and we will only highlight the most important features here. For the present analysis, relevant terms of the low-energy effective Lagrangian in the mass basis read

$$
\mathcal{L}_{\text {eff }}=\varepsilon g_{X} Z_{\mu}^{\prime} \bar{N} \gamma^{\mu} \nu_{L}+\chi c_{W} Z_{\mu}^{\prime} \mathcal{J}_{\text {e.m. }}^{\mu} .
$$

Here, $Z^{\prime}$ is the gauge boson of a new $\mathrm{U}(1)_{\mathrm{X}}$ gauge symmetry with coupling $g_{X}, N$, and $\nu_{L}$ denote, respectively, the new hidden and left-handed standard model (SM) neutrinos, while $\varepsilon$ is the neutrino hidden-neutrino mixing, $c_{W}$ is the cosine of the electroweak (EW) mixing angle [13], $\chi$ the strength of gauge-kinetic mixing of $U(1)_{X}$ and $U(1)_{Y}$ induced by an operator $\mathcal{L}_{\chi}=$ $-\left(s_{\chi} / 2\right) B^{\mu \nu} X_{\mu \nu}[14,15]$, and $\mathcal{J}_{\text {e.m. }}^{\mu}$ the standard electromagnetic current. We only state Eq. (1) for a single flavor of neutrinos $\nu_{L}$ here, which is to be understood as a template for solar electron neutrinos, while the extension to other flavors is straightforward.

From Eq. (1), it is possible to inelastically up-scatter neutrinos to hidden neutrinos on electrons in the XENON1T detector (see Fig. 1) (There is also elastic 


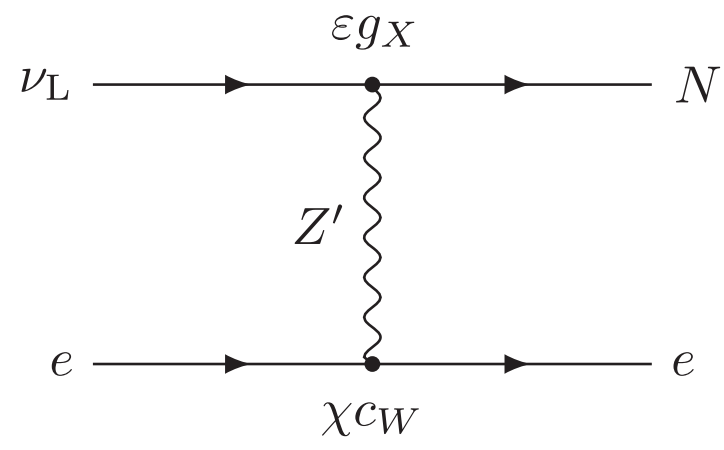

FIG. 1. Feynman diagram for inelastic neutrino to hidden neutrino up-scattering on electrons mediated by a light $Z^{\prime}$. The couplings are generated by active-hidden neutrino mixing on the one side and $U(1)_{X}-U(1)_{Y}$ kinetic mixing on the other.

neutrino-electron scattering, but this is suppressed by another insertion of $\varepsilon$.) Taking $m_{Z^{\prime}} \ll \sqrt{2 m_{e} T}$ and neglecting neutrino masses, the differential cross section for this process is given by (see, e.g., [16])

$$
\frac{\mathrm{d} \sigma_{\nu e \rightarrow N e}}{\mathrm{~d} T}=\frac{\varepsilon^{2} g_{X}^{2} \chi^{2} c_{W}^{2}}{16 \pi m_{e} T^{2}}\left[1+\left(1-\frac{T}{E_{\nu}}\right)^{2}-\frac{m_{e} T}{E_{\nu}^{2}}\right] .
$$

Here $T$ is the electron recoil energy, $E_{\nu}$ the incident neutrino energy, and $m_{e}$ the electron mass. Note the $1 / T^{2}$ enhancement of the differential cross section at low recoil energies. This is akin to the $1 / T$ enhancement in the scattering induced by a neutrino magnetic moment. With more data, it should become possible to discriminate between the energy dependence of our model and, for example, a magnetic moment scattering. Also note that without assuming $m_{Z^{\prime}} \ll \sqrt{2 m_{e} T}$, the actual form of the propagator relevant in Eq. (2) is $\left(2 m_{e} T+m_{Z^{\prime}}^{2}\right)^{2}$, and there is no enhancement for low-energy recoils, which practically rules out an explanation of the excess for mediators heavier than $\sqrt{m_{e} T}$.

The low-energy solar neutrino flux consists essentially of the continuous pp and discrete ${ }^{7} \mathrm{Be}$ flux components [17]

$$
\begin{gathered}
\phi_{\mathrm{pp}}=5.94 \times 10^{10} \mathrm{~cm}^{-2} \mathrm{~s}^{-1}, \\
\phi_{7_{\mathrm{Be}}}=4.86 \times 10^{9} \mathrm{~cm}^{-2} \mathrm{~s}^{-1} .
\end{gathered}
$$

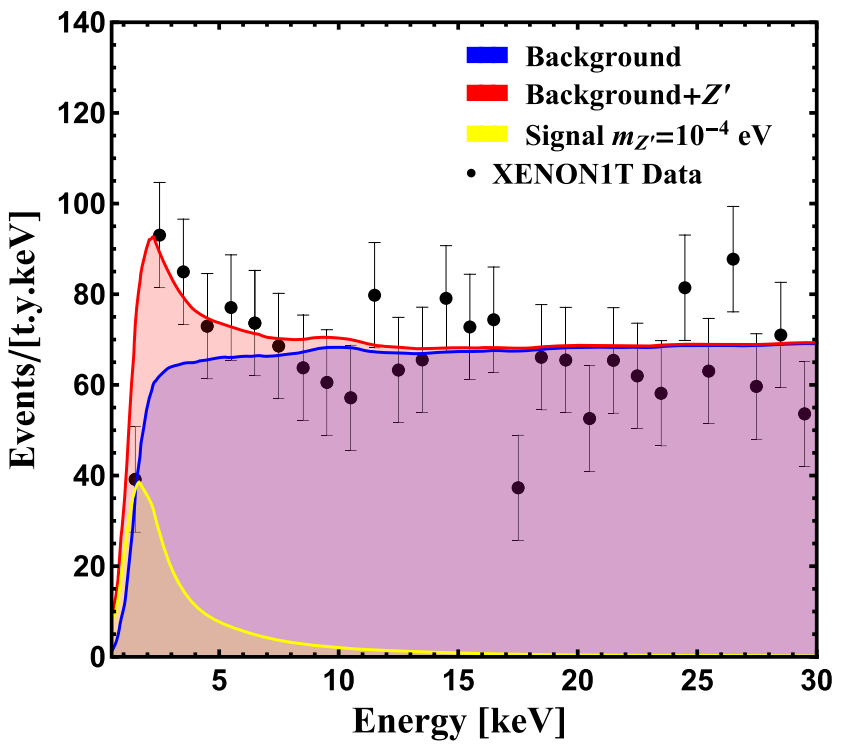

FIG. 2. Event rate for electronic recoils as a function of the electron recoil energy at XENON1T in our model. Data and background taken from [1].

These neutrinos are affected by vacuum-dominated flavor oscillations, resulting in a survival probability of [18]

$$
P_{e e}=\cos ^{4} \theta_{13}\left(1-\frac{1}{2} \sin ^{2} 2 \theta_{12}\right)+\sin ^{4} \theta_{13}
$$

for electron neutrinos arriving at Earth.

To compute our signal prediction, we take into account XENON1T detection and selection efficiency [1] $\epsilon(T)$, as well as the finite detector energy resolution by a gaussian smearing $[19,20]$. Similar to the XENON1T analysis, we use the Free Energy Approximation (FEA)

$$
\frac{\mathrm{d} \sigma_{\mathrm{tot}}}{\mathrm{d} T}=\sum_{i=1}^{54} \Theta\left(T-B_{i}\right) \frac{\mathrm{d} \sigma_{\nu e \rightarrow N e}}{\mathrm{~d} T}
$$

to take into account xenon electron binding energies. At $\mathrm{keV}$ energies, this is a good approximation to more sophisticated computations [21,22]. The differential event rate is then computed by the convolution

$$
\frac{\mathrm{d} N\left(T_{r}\right)}{\mathrm{d} T_{r}}=N_{0} \times t \times \int \mathrm{d} T \mathrm{~d} E_{\nu} \frac{\mathrm{d} \phi\left(E_{\nu}\right)}{\mathrm{d} E_{\nu}} P_{e e} \frac{\mathrm{d} \sigma_{\text {tot }}}{\mathrm{d} T} \Theta\left(\frac{2 E_{\nu}^{2}}{m_{e}+2 E_{\nu}}-T\right) \epsilon\left(T_{r}\right) g^{\text {Gauss }}\left(T_{r}, T\right),
$$

where $T$ and $T_{r}$ are the actual and reconstructed electron recoil energies, respectively.

Fitting this to the observed excess, we obtain Fig. 2. The best fit point has

$$
\varepsilon g_{X} \chi=2.0_{-0.9}^{+0.7} \times 10^{-13} \quad \text { (95\%C.L.) },
$$

and is statistically preferred over the background-only hypothesis by $3 \sigma$.

Note that nothing in our analysis prevents us from considering flavors other than electron neutrinos in Eq. (1). This allows the possibility that also the subdominant nonelectron-flavor solar neutrino flux contributes 
TABLE I. New fields and their charges under SM and new $U(1)_{X}$ gauge symmetry.

\begin{tabular}{lccccc}
\hline \hline Field & $\Phi$ & $N_{1}$ & $N_{2}$ & $S$ & $X_{\mu}$ \\
\hline $\mathrm{SU}(2)_{\mathrm{L}} \times \mathrm{U}(1)_{\mathrm{Y}}$ & $\left(\mathbf{2}, \frac{1}{2}\right)$ & $\varnothing$ & $\varnothing$ & $\varnothing$ & $\varnothing$ \\
$\mathrm{U}(1)_{\mathrm{X}}$ & +1 & +1 & -1 & +1 & 0 \\
\hline \hline
\end{tabular}

to the excess. If the absolute relevant flux changes by a factor $f$, it is straightforward to see that our result in Eq. (8) should be rescaled by $1 / \sqrt{f}$.

We now introduce our complete model in which $\mathcal{L}_{\text {eff }}$ and the parameter region [Eq. (8)] is naturally obtained. New particles and their charges under the new $U(1)_{X}$ gauge symmetry are shown in Table I. We introduce a pair of SM-neutral but $U(1)_{X}$ charged chiral fermions $N_{1,2}$ and two new scalars $\Phi$ and $S$ [25]. New interaction terms for the SM lepton doublet $L=\left(\nu_{L}, e_{L}\right)^{\mathrm{T}}$ are given by

$$
\mathcal{L}_{\text {new }}=-y \bar{L} \tilde{\Phi} N_{1}-M N_{1} N_{2}+\text { H.c. },
$$

where $\tilde{\Phi}:=\mathrm{i} \sigma_{2} \Phi^{*}, y$ is a dimensionless Yukawa coupling, and $M$ has mass dimension one. We only discuss the onegeneration case here, with the extension to three generations of SM leptons and multiple generations of hidden fermions being straightforward. We consider the most general possible scalar potential (cf. [8] for details) and decompose the scalars as

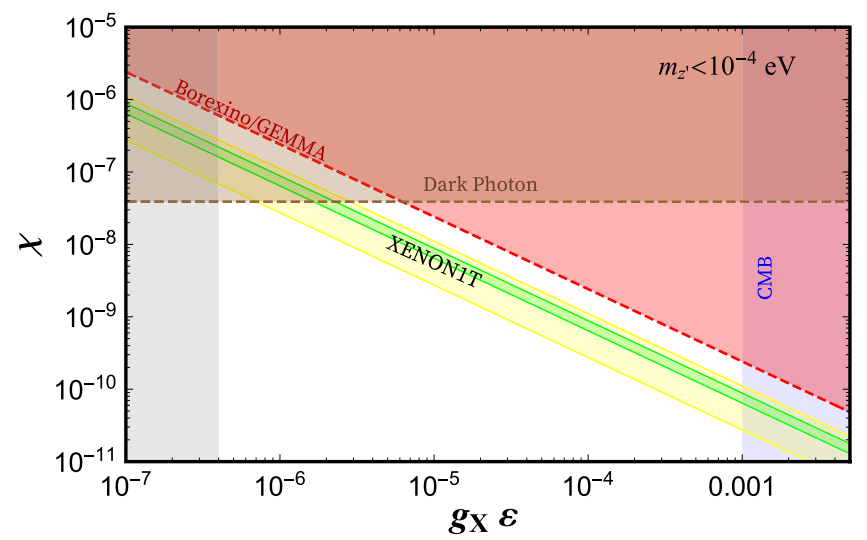

FIG. 3. Constraints on electron-neutrino scattering in the $\varepsilon g_{X}-\chi$ plane. Borexino/GEMMA limits $[23,24]$ and XENON1T signal depends directly on $\varepsilon g_{X} \chi$. Direct limits on $\chi$ arise from dark photon searches [9-11], and they are essentially independent of $m_{Z^{\prime}}$ as long as $m_{Z^{\prime}} \lesssim 10^{-4} \mathrm{eV}$ (they only get weaker below $10^{-9} \mathrm{eV}$ ). Constraints on $\varepsilon^{2} g_{X}$ are given by Eq. (16), with the upper end (blue) excluded by neutrino free streaming during CMB. Their appearance in the plot slides with the exact choice of $\varepsilon$, and we display the widest limit possible for $\varepsilon$ constrained as in Eq. (19).

$$
\begin{gathered}
H=\left(\begin{array}{c}
h^{+} \\
\frac{1}{\sqrt{2}}\left(h+\mathrm{i} a_{h}\right)
\end{array}\right), \quad \Phi=\left(\begin{array}{c}
\phi^{+} \\
\frac{1}{\sqrt{2}}\left(\phi+\mathrm{i} a_{\phi}\right)
\end{array}\right), \\
\text { and } \quad S=\frac{1}{\sqrt{2}}\left(s+\mathrm{i} a_{s}\right) .
\end{gathered}
$$

We assume that all neutral scalars obtain vacuum expectation values (VEVs) $v_{\sigma}:=\langle\sigma\rangle$ for $\sigma=h, \phi, s . v_{h}$ spontaneously breaks EW symmetry, $v_{s}$ breaks $U(1)_{X}$, while $v_{\phi}$ breaks both. We will always assume the hierarchy $v_{h} \ggg v_{s}, v_{\phi}$, which is required by the assumption of a light $Z^{\prime}$. Next to the SM Higgs boson $H$, the physical scalar spectrum consists of a pair of heavy $(>100 \mathrm{GeV})$ charged scalars $\Phi^{ \pm}$, a pair of mass-degenerate heavy neutral scalar and pseudoscalar $\Phi$ and $A\left(\left|m_{\Phi^{ \pm}}-m_{\Phi}\right| \lesssim 120 \mathrm{GeV}\right.$ by electroweak precision), as well as a sub-keV light scalar $h_{S}$, all with very lepton-specific couplings and practically no coupling to quarks.

The photon is exactly the same massless combination of EW bosons as in the SM. The very SM-like $Z$ boson contains a minuscule admixture of the new gauge boson $X$,

$$
Z_{\mu}=c_{X}\left(c_{W} W_{\mu}^{3}-s_{W} B_{\mu}\right)+s_{X} X_{\mu},
$$

with an angle

$$
s_{X} \approx-2 c_{W} \frac{g_{X}}{g_{2}}\left(\frac{v_{\phi}}{v_{h}}\right)^{2} \lll 1 \quad \text { and } \quad c_{X} \approx 1 .
$$

Gauge-kinetic mixing from the operator $\mathcal{L}_{\chi}$ shifts the $Z^{\prime}$ coupling to the SM neutral current only by a negligible amount proportional to $\chi \mathcal{O}\left(m_{Z^{\prime}}^{2} / m_{Z}^{2}\right)$ [31,32] (given $\left.m_{Z^{\prime}} \ll m_{Z}, \chi \ll 1\right)$. However, it will introduce the coupling of $Z^{\prime}$ to the electromagnetic current shown in Eq. (1), which is instrumental for our explanation of the XENON1T excess. The masses of the physical neutral gauge bosons are

$$
m_{Z} \approx \frac{g_{2} v_{h}}{2 c_{W}} \quad \text { and } \quad m_{Z^{\prime}} \approx g_{X} \sqrt{v_{\phi}^{2}+v_{s}^{2}}=: g_{X} \bar{v} .
$$

After $\phi$ assumes its VEV $v_{\phi}$ the Yukawa coupling $y$ will introduce bilinear mixing between SM neutrinos and the new hidden neutrinos. For exactly massless neutrinos, this implies a mixing of $\nu_{L}$ and $\bar{N}_{2}$ by an exact angle

$$
\tan \varepsilon=\left(y v_{\phi}\right) /(\sqrt{2} M) .
$$

Depending on the specific neutrino mass generation mechanism (see, for example, [33-35] for mechanisms compatible with the model) and in particular on whether there is violation of violation of lepton number, this will change slightly for massive neutrinos, in which case there can also be a slight admixture of $\bar{N}_{1}$ and Eq. (15) becomes approximate. In any case, $N$ in Eq. (1) should be 
understood as the resulting hidden-neutrino mass eigenstate, which will have a (Dirac-)mass $M_{N} \approx$ $\left(M^{2}+y^{2} v_{\phi}^{2} / 2\right)^{1 / 2}$. By this mixing, SM neutrinos pick up a coupling to $Z^{\prime}$ from the gauge coupling of $N_{1,2}$. This gives rise to the first term in Eq. (1) but also to a pure SM neutrino $Z^{\prime}$ interaction proportional to $g_{X} \varepsilon^{2}$. For temperatures $T \gg m_{Z^{\prime}}$, while neutrino mixing via $\varepsilon$ is relevant, $Z^{\prime}$ will be effectively massless, giving rise to an induced longrange four-neutrino interaction with the thermally averaged rate $\Gamma \sim \varepsilon^{8} g_{X}^{4} T$. Requiring this rate to not surpass the Hubble rate $H \sim T^{2} / M_{\mathrm{Pl}}$ before recombination, but before today, yields

$$
10^{-8} \lesssim \varepsilon^{2} g_{X} \lesssim 10^{-7}
$$

Clearly, this includes the assumption $m_{Z^{\prime}} \lesssim 1 \mathrm{eV}$. We even focus on the region $m_{Z^{\prime}} \lesssim 10^{-4} \mathrm{eV}$. This is crucial to allow kinetic mixing of up to $\chi \lesssim 5 \times 10^{-8}$, which would be more severely constrained by orders of magnitude for a heavier $m_{Z^{\prime}}$ [9-11]. Parameterizing $m_{Z^{\prime}}=g_{X} \bar{v}$, we can constrain the size of the effective $U(1)_{X}$, breaking VEV $\bar{v}$ to

$$
\bar{v}=\frac{m_{Z^{\prime}}}{g_{X}} \lesssim \varepsilon^{2} \times 10 \times \mathrm{keV} .
$$

This fixes the necessary hierarchy between the relevant scales of the model to

$$
\xi:=\bar{v} / v_{h} \lesssim \varepsilon^{2} \times 4 \times 10^{-8},
$$

where $v_{h}=246 \mathrm{GeV}$ is the SM Higgs VEV. Stabilizing these hierarchies might require tuning in scalar quartic couplings, which would not change any of our conclusions. Combining the requirements Eqs. (8) and (16) and $\chi \lesssim 5 \times 10^{-8}$, we obtain (the lower bound arises from $\left.g_{X}<1\right)$

$$
10^{-4} \lesssim \varepsilon \lesssim 2.5 \times 10^{-2}
$$

This implies we automatically obey constraints arising from violation of the Pontecorvo-Maki-Nakagawa-Sakata unitarity [36,37] or direct search bounds [38-41]. The bounds on $g_{X}$ and $\varepsilon$ also imply a lower bound on $\chi \gtrsim 10^{-10}$, which would be excluded for $m_{Z^{\prime}} \gtrsim 10^{-2} \mathrm{eV}$. This explains why it is not possible to fit the present excess for the parameter region considered in [8]. All constraints in the $\varepsilon g_{X}-\chi$ plane are summarized in Fig. 3. Note that we assume lower scales for $v_{\phi}$ and $v_{s}$ than in [8], but we do not strive to change the relative hierarchy of $v_{\phi}$ and $v_{s}$, parameterized by the angle

$$
\tan \gamma:=v_{\phi} / v_{s} .
$$

This implies that, despite our changes in $m_{Z^{\prime}}, g_{X}$, and $\chi$, other very characteristic details of this model are exactly the same as in [8]. This includes the key signatures $H \rightarrow h_{S} h_{S}$,
$H \rightarrow Z^{\prime} Z^{\prime}, \quad H \rightarrow Z Z^{\prime}$, and $Z \rightarrow Z^{\prime} h_{S}$, whose rates are independent of $m_{Z^{\prime}}, g_{X}$, and $\chi$ because they are fixed by the Goldstone Boson Equivalence. These decays contribute to invisible $H$ and $Z$ decays at potentially observable levels, which already constrain $s_{\gamma} \lesssim 0.2$ [8].

Regarding big bang nucleosynthesis (BBN) constraints, none of the new light states $\left(m_{Z^{\prime}}, h_{S}, N\right)$ was in thermal equilibrium with the SM sufficiently before BBN and between $\mathrm{BBN}$ and recombination, as required by $\mathrm{BBN}$ [42] and CMB constraints [43]. While thermal abundances of the light states are generated by heavy scalar exchange at temperatures above the electroweak scale, any such abundance would be depleted by reheating in the SM-for example, at the QCD phase transition. Still dangerous is the process $e^{+} e^{-}(\nu \bar{\nu}) \leftrightarrow N \bar{N}$ via the $t$-channel $\Phi^{ \pm}(\Phi, A)$ exchange. The absence of this process after the QCD (EW) epoque requires $y \lesssim 6 \times 10^{-3(5)}\left(m_{H^{ \pm}(\Phi)} / 100 \mathrm{GeV}\right)$. Other BBN constraints related to $Z^{\prime}$ coupling to neutrinos do not apply here simply because the new gauge interactions become important only after recombination. The now sizable up-scattering process of Fig. 1 is cosmologically irrelevant.

The leading direct constraint on the effective neutrino$Z^{\prime}$ coupling arises from allowing unperturbed propagation of SN1987A neutrinos through the cosmic neutrino background $(\mathrm{C} \nu \mathrm{B})$ and implies $\varepsilon^{2} g_{X} \lesssim 5 \times 10^{-4}$ [44] (see also [45]). Furthermore, our constraint Eq. (16) already warrants that we are not violating the requirement of free-streaming neutrinos during CMB formation [46]. Laboratory constraints on $m_{Z^{\prime}}$ and $g_{X}$ are not very limiting for light mediators (see references collected in [8]) and become even less relevant here compared to [8] as the effective coupling to neutrinos here is smaller by an order of magnitude.

There are strong constraints on dark photon models and kinetic mixing from stellar cooling if the Higgs mode associated with $U(1)_{X}$ breaking becomes light $[47,48]$. The relevant Higgs mode in our model is $h_{S}$, which has a sub$\mathrm{keV}$-scale mass $m_{h_{S}} \approx \xi v_{h} \sqrt{2 \lambda_{S}}$ but is certainly much heavier than $m_{Z^{\prime}}$. So the stronger bounds of [47] (for the Higgsed case), which assume $m_{h_{S}} \sim m_{Z^{\prime}}$ do not apply at face value. A dedicated analysis in the context of our model would be required, which we expect to give the leading constraint on $g_{X} \chi$ directly. We stress that it is the stellar cooling bounds that matter here, not the direct detection constraints, as the latter can always be avoided if $Z^{\prime}$ decays to (keV energy) neutrinos before arriving at the Earth, which is what generically happens in our model if $m_{Z^{\prime}}>2 m_{\nu}$, i.e., when $Z^{\prime}$ is not a dark matter candidate.

We note there is a parameter region around $m_{Z^{\prime}} \sim 5 \times$ $10^{-4} \mathrm{eV}$ and $\chi \sim 10^{-9}$ (and for $m_{Z^{\prime}}<2 m_{\nu}$ ) where it is not excluded that our $Z^{\prime}$ could make up the entirety of the dark matter (see [11] and references therein). For smaller $m_{Z^{\prime}}$, this possibility is excluded. We stress, though, that nothing in our resolution of the present XENON1T excess depends 
on the possibility of $Z^{\prime}$ being the dark matter. The parameter region $\chi \gtrsim$ few $\times 10^{-9}$ and $m_{Z^{\prime}} \gtrsim 5 \times 10^{-5} \mathrm{eV}$ will be probed by ALPS II [49].

Taking the expression for the mixing angle [Eq. (15)], one can show that

$$
M \lesssim(y / \sqrt{2}) \varepsilon s_{\gamma} \times 10 \times \mathrm{keV}
$$

With the above bounds on $y, \varepsilon$, and $s_{\gamma}$ this would imply $M \lesssim 0.2 \mathrm{eV}$, and $y v_{\phi} \lesssim 8 \times 10^{-3} \mathrm{eV}$. Consequently, neutrino masses will not be a small perturbation but a substantial ingredient in generating the mixing $\varepsilon$, which includes the possibility of lifting $M_{N}$ beyond the above bound. Hence, generating the mixing — unlike in [8]—will depend on the details of the neutrino mass generation mechanism. This might slightly change the valid region of parameters. However, it would not change our conclusion that neutrino up-scattering to hidden states can explain the anomalous excess. On the other hand, this opens the very exciting possibility that we could investigate the neutrino mass generation mechanism by neutrino-electron scattering in XENON1T and complementary experiments, just as in the case where the scattering is due to a neutrino magnetic moment. The exact implications of different neutrino mass generation mechanisms would have to be studied on a caseby-case basis. We also note that, if kinematically allowed by $M_{N}, N$ might decay to $Z^{\prime}$ and neutrinos quickly, i.e., within the detector volume. However, since this is a practically invisible decay, this should not leave an observable signature.

Note that for $m_{\nu, i}>m_{Z^{\prime}}+m_{\nu, j}$, two-body, and for higher masses also three-body, decays of SM neutrinos become possible, depending on the flavor structure of $y$. This fact, and the $Z^{\prime}$-mediated four-neutrino interaction, could substantially modify the cosmic neutrino background. Sufficiently fast decays would render it monogenerational, while the long-range neutrino self-interaction would modify the clustering, even leading to neutrino condensation. In this case, the possible coincidence of $m_{Z^{\prime}} \sim m_{\nu} \sim T_{\mathrm{CMB}}$ could become meaningful for the cosmological "why now?" problem.

In summary, we have outlined a possible explanation for the excess in electronic recoil events observed in XENON1T. In our scenario, the events are caused by inelastic neutrino up-scattering on electrons in the detector medium, induced by the standard solar neutrino flux. Effectively, our explanation is based on a light vector mediator that, on the one hand, has a coupling of SM neutrinos to hidden neutrinos and, on the other hand, couples to the electromagnetic current. More specifically, these couplings are understood to originate from neutrino mass mixing and gauge-kinetic mixing between a new $U(1)_{X}$ gauge symmetry with the SM hypercharge. This model could be distinguished from other explanations of the XENON1T excess by the $1 / T^{2}$ recoil-energy dependence of the differential cross section.

We have also presented an explicit gauge invariant, renormalizable, and UV-complete model that realizes this explanation of the XENON1T excess without conflicting with observational constraints. The model would also lead to long-range neutrino self-interactions that could substantially modify the appearance of the cosmic neutrino background. More accessible signatures of the explicit model are new invisible Higgs and $Z$ decays and the presence of leptophilic charged and neutral scalars with masses $\mathcal{O}(100 \mathrm{GeV})$, which all could be searched for with the LHC and future colliders. Sterile neutrinos are required, and they should mix with the SM neutrinos with an angle $\varepsilon>10^{-4}$. The parameter space of the model can further be tested by searches for dark vectors and kinetic mixing, which are being actively pursued — at ALPS II [49], for example. Leading limits on electron-neutrino scattering are set by the TEXONO [50], Borexino [23], and GEMMA [24] experiments [51], while complementary regions of parameter space will also be probed by electron recoils at ongoing reactor neutrino experiments $[16,52]$ like CONUS [53], CONNIE [54], or $\nu$-cleus [55].

If the excess and our explanation holds up, this may open the exciting possibility of using neutrino-electron scattering to learn more about the mechanism behind neutrino mass generation and potentially strong neutrino self-interactions.

We thank Xunjie $\mathrm{Xu}$ and Manfred Lindner for useful conversations.

Note added.-After our paper was submitted to, but before it appeared on the arXiv, Refs. [56-58] appeared which also treat the XENON1T excess (See also Ref. [59] which was submitted before our paper appeared). Especially [57] also considers neutrino-electron scattering by a light vector mediator, despite in a somewhat effective picture without complete model. Our results are consistent where overlapping, but we stress that mediator masses heavier than $m_{Z^{\prime}} \gtrsim 1 \mathrm{eV}$ are excluded by Dark Photon constraints; which unavoidably brings long-range neutrino self-interaction into focus.

*Corresponding author. andreas.bally@mpi-hd.mpg.de

Corresponding author.

sudip.jana@mpi-hd.mpg.de

"Corresponding author. trautner@mpi-hd.mpg.de

[1] E. Aprile et al., Observation of excess electronic recoil events in XENON1T, arXiv:2006.09721.

[2] F. Takahashi, M. Yamada, and W. Yin, preceding Letter, XENON1T Anomaly from Anomaly-Free ALP Dark Matter and its Implications for Stellar Cooling Anomaly, Phys. Rev. Lett. 125, 161801 (2020). 
[3] K. Kannike, M. Raidal, H. Veerme, A. Strumia, and D. Teresi, Dark matter and the XENON1T electron recoil excess, arXiv:2006.10735.

[4] M. Giannotti, I. G. Irastorza, J. Redondo, A. Ringwald, and K. Saikawa, Stellar recipes for axion hunters, J. Cosmol. Astropart. Phys. 10 (2017) 010.

[5] A. Córsico, L. Althaus, M. M. Bertolami, S. Kepler, and E. García-Berro, Constraining the neutrino magnetic dipole moment from white dwarf pulsations, J. Cosmol. Astropart. Phys. 08 (2014) 054.

[6] S. Arceo-Daz, K. P. Schrder, K. Zuber, and D. Jack, Constraint on the magnetic dipole moment of neutrinos by the tip-RGB luminosity in $\omega$-Centauri, Astropart. Phys. 70, 1 (2015).

[7] S. A. Daz, K.-P. Schrder, K. Zuber, D. Jack, and E. E. B. Barrios, Constraint on the axion-electron coupling constant and the neutrino magnetic dipole moment by using the tip-RGB luminosity of fifty globular clusters, arXiv:1910.10568.

[8] M. Berbig, S. Jana, and A. Trautner, The Hubble tension and a renormalizable model of gauged neutrino self-interactions, arXiv:2004.13039.

[9] J. Redondo, Atlas of solar hidden photon emission, J. Cosmol. Astropart. Phys. 07 (2015) 024.

[10] T. Gherghetta, J. Kersten, K. Olive, and M. Pospelov, Evaluating the price of tiny kinetic mixing, Phys. Rev. D 100, 095001 (2019).

[11] M. Fabbrichesi, E. Gabrielli, and G. Lanfranchi, The dark photon, arXiv:2005.01515.

[12] L. Vecchi, Light sterile neutrinos from a late phase transition, Phys. Rev. D 94, 113015 (2016).

[13] We abbreviate the trigonometric functions of all angles by $\sin \theta_{i} \equiv s_{i}, \cos \theta_{i} \equiv c_{i}$, and $\tan \theta_{i} \equiv t_{i}$ in this Letter.

[14] P. Galison and A. Manohar, Twi Z's or not two Z's?, Phys. Lett. 136B, 279 (1984).

[15] B. Holdom, Two U(1)'s and epsilon charge shifts, Phys. Lett. 166B, 196 (1986).

[16] M. Lindner, F. S. Queiroz, W. Rodejohann, and X.-J. Xu, Neutrino-electron scattering: general constraints on $\mathrm{Z}$ and dark photon models, J. High Energy Phys. 05 (2018) 098.

[17] J. N. Bahcall and C. Pena-Garay, Solar models and solar neutrino oscillations, New J. Phys. 6, 63 (2004).

[18] W. Haxton, R. Hamish Robertson, and A. M. Serenelli, Solar neutrinos: Status and prospects, Annu. Rev. Astron. Astrophys. 51, 21 (2013).

[19] E. Aprile et al. (XENON Collaboration), The XENON1T dark matter experiment, Eur. Phys. J. C 77, 881 (2017).

[20] E. Aprile et al. (XENON Collaboration), Energy resolution and linearity in the $\mathrm{keV}$ to $\mathrm{MeV}$ range measured in XENON1T, Eur. Phys. J. C 80, 785 (2020).

[21] J.-W. Chen, H.-C. Chi, C. P. Liu, and C.-P. Wu, Low-energy electronic recoil in xenon detectors by solar neutrinos, Phys. Lett. B 774, 656 (2017).

[22] C.-C. Hsieh, L. Singh, C.-P. Wu, J.-W. Chen, H.-C. Chi, C.-P. Liu, M. K. Pandey, and H. T. Wong, Discovery potential of multiton xenon detectors in neutrino electromagnetic properties, Phys. Rev. D 100, 073001 (2019).

[23] M. Agostini et al. (BOREXINO Collaboration), Comprehensive measurement of $p p$-chain solar neutrinos, Nature (London) 562, 505 (2018).
[24] A. Beda, V. B. Brudanin, V. G. Egorov, D. V. Medvedev, V.S. Pogosov, E. A. Shevchik, M. V. Shirchenko, A. S. Starostin, and I. V. Zhitnikov, Gemma experiment: The results of neutrino magnetic moment search, Phys. Part. Nucl. Lett. 10, 139 (2013).

[25] Similar models, albeit in a completely different range of parameters, have been conceived in [26-28] (see also [29,30] for similar but somewhat incomplete models).

[26] Y. Farzan and J. Heeck, Neutrinophilic nonstandard interactions, Phys. Rev. D 94, 053010 (2016).

[27] Y. Farzan and M. Tortola, Neutrino oscillations and non-standard interactions, Front. Phys. 6, 10 (2018).

[28] P. B. Denton, Y. Farzan, and I. M. Shoemaker, Activating the fourth neutrino of the $3+1$ scheme, Phys. Rev. D 99, 035003 (2019).

[29] P. Ballett, M. Hostert, and S. Pascoli, Dark neutrinos and a three portal connection to the Standard Model, Phys. Rev. D 101, 115025 (2020).

[30] P. Ballett, M. Hostert, and S. Pascoli, Neutrino masses from a dark neutrino sector below the electroweak scale, Phys. Rev. D 99, 091701 (2020).

[31] M. Baumgart, C. Cheung, J. T. Ruderman, L.-T. Wang, and I. Yavin, Non-Abelian dark sectors and their collider signatures, J. High Energy Phys. 04 (2009) 014.

[32] K. Babu, A. Friedland, P. Machado, and I. Mocioiu, Flavor gauge models below the Fermi scale, J. High Energy Phys. 12 (2017) 096.

[33] R. N. Mohapatra, New contributions to neutrinoless doublebeta decay in supersymmetric theories, Phys. Rev. D 34, 3457 (1986).

[34] E. Bertuzzo, S. Jana, P. A. N. Machado, and R. Z. Funchal, Neutrino masses and mixings dynamically generated by a light dark sector, Phys. Lett. B 791, 210 (2019).

[35] P. S. B. Dev and A. Pilaftsis, Minimal radiative neutrino mass mechanism for inverse seesaw models, Phys. Rev. D 86, 113001 (2012).

[36] S. Antusch and O. Fischer, Non-unitarity of the leptonic mixing matrix: Present bounds and future sensitivities, J. High Energy Phys. 10 (2014) 094.

[37] E. Fernandez-Martinez, J. Hernandez-Garcia, and J. Lopez-Pavon, Global constraints on heavy neutrino mixing, J. High Energy Phys. 08 (2016) 033.

[38] A. de Gouva and A. Kobach, Global constraints on a heavy neutrino, Phys. Rev. D 93, 033005 (2016).

[39] D. A. Bryman and R. Shrock, Improved constraints on sterile neutrinos in the $\mathrm{MeV}$ to $\mathrm{GeV}$ mass range, Phys. Rev. D 100, 053006 (2019).

[40] D. A. Bryman and R. Shrock, Constraints on sterile neutrinos in the $\mathrm{MeV}$ to $\mathrm{GeV}$ mass range, Phys. Rev. D 100, 073011 (2019).

[41] P. D. Bolton, F. F. Deppisch, and P. Bhupal Dev, Neutrinoless double beta decay versus other probes of heavy sterile neutrinos, J. High Energy Phys. 03 (2020) 170.

[42] B. D. Fields, K. A. Olive, T.-H. Yeh, and C. Young, Big-bang nucleosynthesis after Planck, J. Cosmol. Astropart. Phys. 03 (2020) 010.

[43] N. Aghanim et al. (Planck Collaboration), Planck 2018 results. VI. Cosmological parameters, arXiv:1807.06209. 
[44] E. W. Kolb and M. S. Turner, Supernova SN 1987a and the secret interactions of neutrinos, Phys. Rev. D 36, 2895 (1987).

[45] R. Konoplich and M. Khlopov, Constraints on triplet Majoron model due to observations of neutrinos from stellar collapse, Sov. J. Nucl. Phys. 47, 565 (1988).

[46] S. Hannestad and G. G. Raffelt, Constraining invisible neutrino decays with the cosmic microwave background, Phys. Rev. D 72, 103514 (2005).

[47] H. An, M. Pospelov, and J. Pradler, Dark Matter Detectors as Dark Photon Helioscopes, Phys. Rev. Lett. 111, 041302 (2013).

[48] J. Redondo and G. Raffelt, Solar constraints on hidden photons re-visited, J. Cosmol. Astropart. Phys. 08 (2013) 034.

[49] R. Bhre et al., Any light particle search II-Technical design report, J. Instrum. 8, T09001 (2013).

[50] M. Deniz et al. (TEXONO Collaboration), Constraints on nonstandard neutrino interactions and unparticle physics with neutrino-electron scattering at the Kuo-Sheng nuclear power reactor, Phys. Rev. D 82, 033004 (2010).

[51] S. Bilmis, I. Turan, T. Aliev, M. Deniz, L. Singh, and H. Wong, Constraints on dark photon from neutrino-electron scattering experiments, Phys. Rev. D 92, 033009 (2015).

[52] J. B. Dent, B. Dutta, D. Kim, S. Liao, R. Mahapatra, K. Sinha, and A. Thompson, New Directions for Axion
Searches via Scattering at Reactor Neutrino Experiments, Phys. Rev. Lett. 124, 211804 (2020).

[53] C. Buck et al., A novel experiment for coherent elastic neutrino nucleus scattering: CONUS, J. Phys. Conf. Ser. 1342, 012094 (2020).

[54] A. Aguilar-Arevalo et al. (CONNIE Collaboration), The CONNIE experiment, J. Phys. Conf. Ser. 761, 012057 (2016).

[55] R. Strauss et al., The $\nu$-cleus experiment: A gram-scale fiducial-volume cryogenic detector for the first detection of coherent neutrino-nucleus scattering, Eur. Phys. J. C 77, 506 (2017).

[56] B. Fornal, P. Sandick, J. Shu, M. Su, and Y. Zhao, this issue, Boosted Dark Matter Interpretation of the XENON1T Excess, Phys. Rev. Lett. 125, 161804 (2020).

[57] G. Alonso-lvarez, F. Ertas, J. Jaeckel, F. Kahlhoefer, and L. Thormaehlen, Hidden Photon Dark Matter in the Light of XENON1T and Stellar Cooling, arXiv:2006.11243.

[58] C. Boehm, D. G. Cerdeno, M. Fairbairn, P. A. Machado, and A.C. Vincent, Light new physics in XENON1T, arXiv:2006.11250

[59] N. F. Bell, J. B. Dent, B. Dutta, S. Ghosh, J. Kumar, and J. L. Newstead, following Letter, Explaining the XENON1T Excess with Luminous Dark Matter, Phys. Rev. Lett. 125, 161803 (2020). 